\title{
Propofol induces the ferroptosis of colorectal cancer cells by downregulating STAT3 expression
}

\author{
$\mathrm{XINING} \mathrm{ZHAO}^{1}$ and FEI CHEN ${ }^{2}$ \\ ${ }^{1}$ Department of Anesthesia, Zhongshan Hospital, Fudan University, Shanghai 200032; ${ }^{2}$ Department of Anesthesiology, \\ Mindong Hospital Affiliated to Fujian Medical University, Fu'an, Fujian 355000, P.R. China
}

Received February 25, 2021; Accepted July 22, 2021

DOI: $10.3892 / 01.2021 .13028$

\begin{abstract}
Propofol is a commonly used intravenous anesthetic agent that can also suppress the proliferation of various human cancer types, including colorectal cancer (CRC). The present study aimed to investigate whether propofol could induce the ferroptosis of CRC cells by regulating signal transducer and activator of transcription 3 (STAT3). STAT3 expression in normal and CRC tissues was measured. Human normal colonic epithelial NCM460 cells and human CRC SW480 cells were exposed to different concentrations of propofol and then cell viability was detected. SW480 cells were transfected with a vector overexpressing STAT3 and treated with propofol, and the cell viability, colony formation, cell proliferation, iron level, ROS production and ferroptosis of these cells and control cells were evaluated. Overall, the results showed that STAT3 was highly expressed in CRC tissues. Propofol exerted no marked effect on NCM460 cell viability, but inhibited SW480 cell viability in a concentration-dependent manner. Meanwhile, STAT3 was downregulated by propofol in a concentration-dependent manner. Propofol also inhibited CRC cell proliferation and colony formation, and enhanced cellular iron and ROS levels. Additionally, the expression of proteins involved in ferroptosis was also altered by propofol, including the upregulation of CHAC1 and PTGS2 expression in CRC cells, and the inhibition of GPX4 expression. However, STAT3 overexpression blocked the effect of propofol on CRC cells. In conclusion, propofol may trigger the ferroptosis of CRC cells by downregulating STAT3 expression.
\end{abstract}

\section{Introduction}

Colorectal cancer (CRC) is a common malignant tumor of the gastrointestinal tract that is characterized by high incidence

Correspondence to: Dr Fei Chen, Department of Anesthesiology, Mindong Hospital Affiliated to Fujian Medical University, 89 Heshan Road, Fu'an, Fujian 355000, P.R. China

E-mail: drchenfei@126.com

Key words: colorectal cancer, ferroptosis, propofol, signal transducer and activator of transcription 3 and mortality rates; as developing countries continue to progress, the incidence of colorectal cancer is estimated to increase to 2.5 million new cases worldwide by $2035(1,2)$. The early symptoms of CRC may not be obvious; however, as the cancer progresses, symptoms such as the presence of blood in the stool, diarrhea, changes in bowel habits, local abdominal pain and anemia appear, which seriously affect the health and quality of life of the patients (2). To improve the treatment efficacy of CRC, chemotherapy, radiation and surgical resection are often used in combination. However, conventional treatments are usually accompanied by severe side effects, toxicity or chemotherapy resistance (3). Therefore, identifying novel drugs that are effective in CRC and have a favorable toxicity profile remains a priority for researchers.

As well as being a commonly used intravenous anesthetic for clinical anesthesia, 2,6-diisopropyl phenol (propofol) has been found to possess considerable non-anesthetic properties, including being able to prevent nausea and vomiting, activate $\gamma$-aminobutyric acid receptors to enhance analgesia, regulate nitric oxide synthesis, and exert antioxidant, neuroprotective, anti-anxiety, immunoregulatory and anti-platelet aggregation effects (4). Notably, propofol has also been found to inhibit the proliferation and migration of tumor cells, promote tumor cell apoptosis and enhance the sensitivity to chemotherapeutic drugs by modulating microRNA expression, gene transcription, apoptosis and immune system function (5). A retrospective cohort study reported that propofol anesthesia during CRC surgery was associated with an improved survival rate, irrespective of the Tumor-Node-Metastasis stage (6). Another study demonstrated that propofol suppressed CRC tumorigenesis by promoting cell apoptosis (7). The aforementioned data indicate the beneficial role of propofol in inhibiting CRC tumorigenesis; however, to the best of our knowledge, the underlying mechanism remains poorly understood.

Inducing tumor cell death is the main strategy for clinical cancer treatment and most anticancer drugs eliminate tumor cells by triggering apoptosis and cell necrosis (8). However, owing to the acquired or inherent resistance of cancer cells to apoptosis and necrosis, the effectiveness of inducing tumor cell death via these mechanisms is limited (9). Ferroptosis, a type of cell death that is different to apoptosis and necrosis, has recently attracted widespread attention due to its observed effectiveness in killing cancer cells (10). Ferroptosis is an 
iron-dependent and reactive oxygen species (ROS)-associated type of cell death, characterized by the accumulation of ROS and the inactivation of the cellular antioxidant glutathione (GSH), which leads to redox dysregulation (11). A large number of studies reported that the induction of ferroptosis successfully killed tumor cells and inhibited tumor growth. In $\mathrm{CRC}$, the activation of ferroptosis has also been suggested to represent a promising therapeutic option and the underlying mechanisms involved have been extensively reported (12-14). Previous studies reported that propofol induced cancer cell death via ROS-mediated apoptosis $(15,16)$. Therefore, it was hypothesized that propofol may inhibit CRC tumorigenesis via the induction of ferroptosis-mediated cell death.

According to the prediction results from the Search Tools for Interactions of Chemicals (STITCH) database, propofol regulates STAT3, which is known to be activated in numerous types of cancer, including CRC, where it promotes cancer progression (17). In addition, STAT3 has also been found to regulate ferroptosis (18). The present study investigated whether propofol can trigger ferroptosis by downregulating STAT3 expression, thereby inhibiting CRC tumorigenesis.

\section{Materials and methods}

Patient studies. A total of 12 paired CRC cancerous and paracancerous tissues were collected from patients who underwent a radical resection of colorectal carcinoma at Zhongshan Hospital (Shanghai, China) between May 2020 and August 2020. Patients were aged 18-90 years (mean age, 73.8 years), with a male to female ratio of $1: 1$, and were divided into 12 cases of cancerous tissue and 12 cases of paracancerous tissue. All the patients provided written, informed consent and the study was approved by the Ethical Committee of Zhongshan Hospital (approval no. B2016-014). Exclusion criteria were as follows: Patients with combined autoimmune diseases; a history of other malignant tumors; a history of preoperative radiotherapy; patients under 18 years of age; or older patients who could not cooperate with the investigation.

Cell culture and treatment. The human normal colonic epithelial cell line, NCM460, and the human CRC cell line, SW480, were purchased from The Cell Bank of the Type Culture Collection of The Chinese Academy of Sciences. Cells were cultured in DMEM (Gibco; Thermo Fisher Scientific, Inc.) supplemented with 10\% FBS (Gibco; Thermo Fisher Scientific, Inc.), and maintained in a humidified atmosphere at $37^{\circ} \mathrm{C}$ containing $5 \% \mathrm{CO}_{2}$. Propofol (MilliporeSigma) was diluted in DMSO and then in culture medium to specific concentrations. NCM460 and SW480 cells were treated with different concentrations of propofol $(12.5,25$ and $50 \mu \mathrm{m})$ for $48 \mathrm{~h}$ at room temperature, and cells receiving no treatment were considered as the controls.

Cell transfection. The human STAT3 cDNA sequence was synthesized by GenScript and inserted into a pcDNA3.1 vector (Invitrogen; Thermo Fisher Scientific, Inc.). SW480 cells (70-90\% confluence) were seeded into 6-well plates and transfected with STAT3 gene recombinant vector pcDNA3.1-STAT3 (pc-STAT3; $10 \mathrm{nM}$ ) or pcDNA3.1 blank vector (pcDNA3.1; $10 \mathrm{nM}$ ) using Lipofectamine ${ }^{\circledR} 2000$ reagent
(Invitrogen; Thermo Fisher Scientific, Inc.) according to the manufacturer's protocol. Following transfection, cells were subjected to propofol treatment. Following incubation at $37^{\circ} \mathrm{C}$ for $5 \mathrm{~h}$, untransfected SW480 cells and SW480 cells transfected with pc-STAT3 or pcDNA3.1 were exposed to DMEM or medium supplemented with $50 \mu \mathrm{m}$ propofol. The control group was SW480 cells exposed to control medium without transfection. Subsequent experimentation was conducted within $48 \mathrm{~h}$.

MTT assay. An MTT assay was used to determine cell viability. Briefly, NCM460 and SW480 cells were seeded at a density of $4 \times 10^{3}$ cells/well into 96 -well plates. Following incubation for $48 \mathrm{~h}, 10 \mu \mathrm{l}$ MTT solution (Beyotime Institute of Biotechnology) was added to each well and further incubated for $4 \mathrm{~h}$. The cell culture medium was subsequently discarded and $150 \mu \mathrm{l}$ DMSO was added to the cells. The absorbance was measured at a wavelength of $570 \mathrm{nM}$ using a high-throughput universal microplate assay (BMG Labtech $\mathrm{GmbH}$ ).

Colony formation assay. For the colony formation assay, $1 \times 10^{3}$ SW480 cells from the control, propofol, propofol + pcDNA3.1 and propofol + pc-STAT3 groups were plated into $3.5-\mathrm{cm}$ dished and maintained in media supplemented with $10 \%$ FBS to allow colony formation, with the medium being replaced every 4 days. Following $\sim 2$ weeks of incubation, the colonies were fixed with methanol for $15 \mathrm{~min}$ at room temperature and stained with $0.1 \%$ crystal violet for $15 \mathrm{~min}$ at room temperature. The stained colonies with $>50$ cells were subsequently manually counted.

Lipid peroxidation [thiobarbituric acid reactive substances (TBARS)]. Lipid peroxidation was detected by a TBARS kit assay (cat. no. STA-330; OxiSelect ${ }^{\mathrm{TM}}$; Cell Biolabs Inc.) using RIPA lysis buffer (Beyotime Institute of Biotechnology) containing SW480 cells $\left(1 \times 10^{7} / \mathrm{ml}\right)$. SW480 cells treated with propofol and/or transfected with pc-STAT3 were harvested and transferred to a centrifuge tube, then centrifuged for $10 \mathrm{~min}$ at $1,000 \mathrm{x} \mathrm{g}$ at room temperature. The process was conducted according to a previous study (19). The absorbance of the aforementioned solution was detected by a microplate reader at $535 \mathrm{nM}$.

Measurement of total iron and $\mathrm{Fe}^{2+}$ levels. An Iron assay kit (cat. no. MAK025; MilliporeSigma) was used to measure total iron and $\mathrm{Fe}^{2+}$ levels in cells. A total of $2 \times 10^{6} \mathrm{SW} 480$ cells were quickly homogenized in 4-10X Iron Assay buffer. The sample was subsequently centrifuged at $13,000 \mathrm{x}$ g for $10 \mathrm{~min}$ at $4^{\circ} \mathrm{C}$ to remove the insoluble materials. To measure total iron, $5 \mu 1$ iron reducer was added to each sample well to reduce $\mathrm{Fe}^{3+}$ to $\mathrm{Fe}^{2+}$ and a horizontal shaker or pipette was used to mix the samples thoroughly, prior to incubation at room temperature in the dark for $30 \mathrm{~min}$. To measure ferrous iron levels, $5 \mu \mathrm{l}$ assay buffer was added to the samples in one set of wells and $5 \mu 1$ iron reducer was added to the other set of wells. Next, $100 \mu \mathrm{l}$ iron probe was added to each well containing the standard or test samples. Samples were mixed using a horizontal shaker or by pipetting, and were then incubated for $60 \mathrm{~min}$ at room temperature in the dark. Finally, the absorbance was measured at a wavelength of $593 \mathrm{nM}$. 
ROS detection. Changes in intracellular ROS levels were measured using a 2',7'-dichlorofluorescein diacetate (DCFDA)-ROS kit (cat. no. 113851; Abcam). Briefly, SW480 cells were plated into a $6-\mathrm{cm}$ culture dish at a density of $1 \times 10^{5}$ cells $/ \mathrm{ml}$ and allowed to attach overnight. Cells overexpressing STAT3 or untransfected cells were subsequently incubated with control media or propofol for $48 \mathrm{~h}$. The cells were then washed with Hanks' balanced salt solution and incubated with DCFDA at $37^{\circ} \mathrm{C}$ for $45 \mathrm{~min}$. The level of ROS was detected by an inverted fluorescence microscope. Quantitative analysis was performed using ImageJ software (version 1.52r; National Institutes of Health).

Reverse transcription-quantitative PCR. Total RNA was extracted from SW480 cells and NCM460 cells using TRIzol ${ }^{\circledR}$ reagent (Invitrogen; Thermo Fisher Scientific, Inc.), according to the manufacturer's protocol. Total RNA was reverse-transcribed into cDNA at $42^{\circ} \mathrm{C}$ for $30 \mathrm{~min}$ using a PrimeScript ${ }^{\mathrm{TM}}$ RT reagent kit (Takara Biotechnology Co., Ltd.). qPCR was subsequently performed using a SYBR-Green Master PCR mix (Applied Biosystems; Thermo Fisher Scientific, Inc.) on a TP800 Thermal Cycler Dice ${ }^{\mathrm{TM}}$ Real Time system (Takara Biotechnology Co., Ltd.). The following thermocycling conditions were used: Initial denaturation at $95^{\circ} \mathrm{C}$ for $10 \mathrm{~min}$, followed by $95^{\circ} \mathrm{C}$ for $15 \mathrm{sec}$ and $60^{\circ} \mathrm{C}$ for $1 \mathrm{~min}$ for 40 cycles, with a final extension at $72^{\circ} \mathrm{C}$ for $10 \mathrm{~min}$. The primer sequences used for the qPCR were as follows: STAT3 forward, 5'-CATCCTGAAGCTGACCCA GG-3' and reverse, 5'-TCCTCACATGGGGGAGGTAG-3'; and GAPDH forward, 5'-GAAAGCCTGCCGGTGACTAA-3' and reverse, 5'-TTCCCGTTCTCAGCCTTGAC-3'. Relative STAT3 mRNA expression levels were quantified using the $2^{-\Delta \Delta \mathrm{Cq}}$ method (20) and normalized to GAPDH, the internal control.

Western blotting. Total protein was extracted from SW480 cells and NCM460 cells using RIPA lysis buffer (Beyotime Institute of Biotechnology) and quantified using a BCA assay kit (Beyotime Institute of Biotechnology). A total of $30 \mu \mathrm{g}$ protein/lane was separated using $12 \%$ gels via SDS-PAGE and subsequently transferred onto PVDF membranes. After blocking with 5\% skimmed milk for $2 \mathrm{~h}$ at room temperature, the membranes were then incubated with the following primary antibodies overnight at $4^{\circ} \mathrm{C}$ : Anti-STAT3 (cat. no. ab68153; dilution, 1:1,000; Abcam), anti-Ki67 (cat. no. ab92742; dilution, 1:5,000; Abcam), anti-proliferating cell nuclear antigen (PCNA) (cat. no. ab92552; dilution, 1:1,000; Abcam), anti-cation transport regulator homolog 1 (CHAC1) (cat. no. \#PA5-103719; dilution, 1:1,000; Invitrogen; Thermo Fisher Scientific, Inc.), anti-prostaglandin-endoperoxide synthase 2 (PTGS2) (cat. no. ab52237; dilution, 1:1,000; Abcam), anti-glutathione peroxidase 4 (GPX4) (cat. no. ab125066; dilution, 1:1,000; Abcam) and anti-GAPDH (cat. no. ab9485; dilution, 1:2,500; Abcam). Following the primary antibody incubation, the membranes were incubated with a goat anti-rabbit HRP-conjugated antibody (cat. no. ab6721; dilution, 1:2,000; Abcam) for $1 \mathrm{~h}$ at room temperature. The protein bands were imaged on a Tanon Chemiluminescence Imaging system with Pierce $^{\mathrm{TM}}$ ECL Western regent (cat. no. 32209; Thermo Fisher Scientific, Inc.). The intensity of the bands was semi-quantified using ImageJ software (version 1.52r; National Institutes of Health).

Statistical analysis. Statistical analysis was performed using GraphPad Prism 6 software (GraphPad Software, Inc.). Data are presented as the mean \pm standard deviation. All experiments were repeated in triplicate. Statistical differences were determined using a paired or unpaired Student's t-test between two groups, or one-way ANOVA followed by Tukey's post hoc test among multiple groups. $\mathrm{P}<0.05$ was considered to indicate a statistically significant difference.

\section{Results}

Propofol upregulates STAT3 expression in SW480 CRC cell lines. To determine the role of STAT3 in CRC, the mRNA expression levels of STAT3 in control normal and CRC tissues were analyzed. Compared with those in the control tissues, STAT3 expression levels were significantly upregulated in the CRC tissues, indicating the potential role of STAT3 in CRC (Fig. 1A). The mRNA and protein expression levels of STAT3 in SW480 cells were all higher than those in NCM460 cells (Fig. 1B and C). The chemical structural formula of propofol is shown as Fig. 1D. Both cell lines were exposed to $0,12.5,25$ or $50 \mu \mathrm{m}$ propofol for $48 \mathrm{~h}$, and then cell viability was analyzed using an MTT assay. As shown in Fig. 1E, propofol treatment did not significantly alter NCM460 cell viability, but decreased CRC SW480 cell viability. The expression levels of STAT3 in SW480 cells following propofol treatment were also analyzed. As shown in Fig. 1F and G, the mRNA and protein expression levels of STAT3 were significantly downregulated by propofol treatment in a concentration-dependent manner. These data suggested that propofol may decrease CRC cell viability by downregulating STAT3 expression. As $50 \mu \mathrm{m}$ propofol exhibited the highest inhibitory effect on the expression of STAT3, this dose was selected for use in the following experiments. The dose chosen was the dose where the cell survival level was $>50 \%$. If the dose were greater than the $50 \%$ maximal inhibitory concentration, it would lead to excessive cell death and would not be conducive to the cell proliferation observed later in the study.

Propofol inhibits CRC cell proliferation and colony formation, while STAT3 overexpression inhibits these effects. pcDNA3.1-STAT3 overexpression vectors were transfected into SW480 cells to overexpress STAT3. The data shown in Fig. 2A and B revealed that STAT3 was successfully overexpressed in the cells, as demonstrated by significantly upregulated mRNA and protein expression levels of STAT3 in SW480 cells transfected with the overexpression vector. Untreated and SW480 cells overexpressing STAT3 were exposed to control medium or medium supplemented with $50 \mu \mathrm{m}$ propofol, then cell proliferation and the colony formation ability were assessed. The results demonstrated that propofol treatment markedly inhibited cell proliferation, while STAT3 overexpression promoted cell proliferation (Fig. 2C). The results of the colony formation assay demonstrated that the inhibitory effect of propofol on cell colony formation was also reversed by STAT3 overexpression (Fig. 2D). Similarly, propofol treatment downregulated the expression levels of 
A

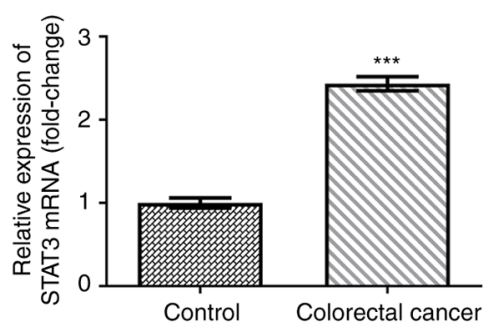

B

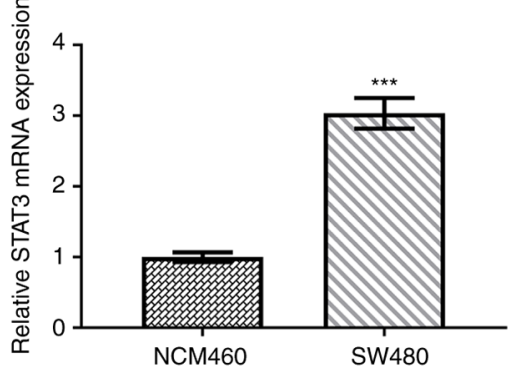

D

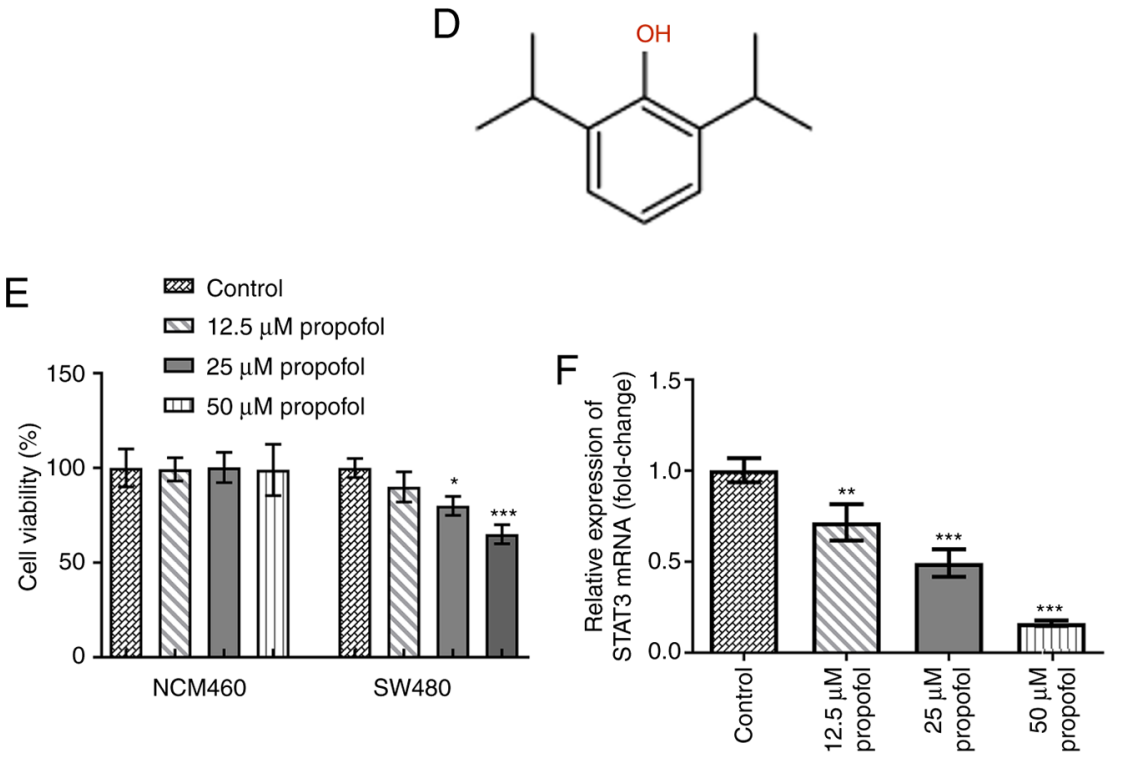

E

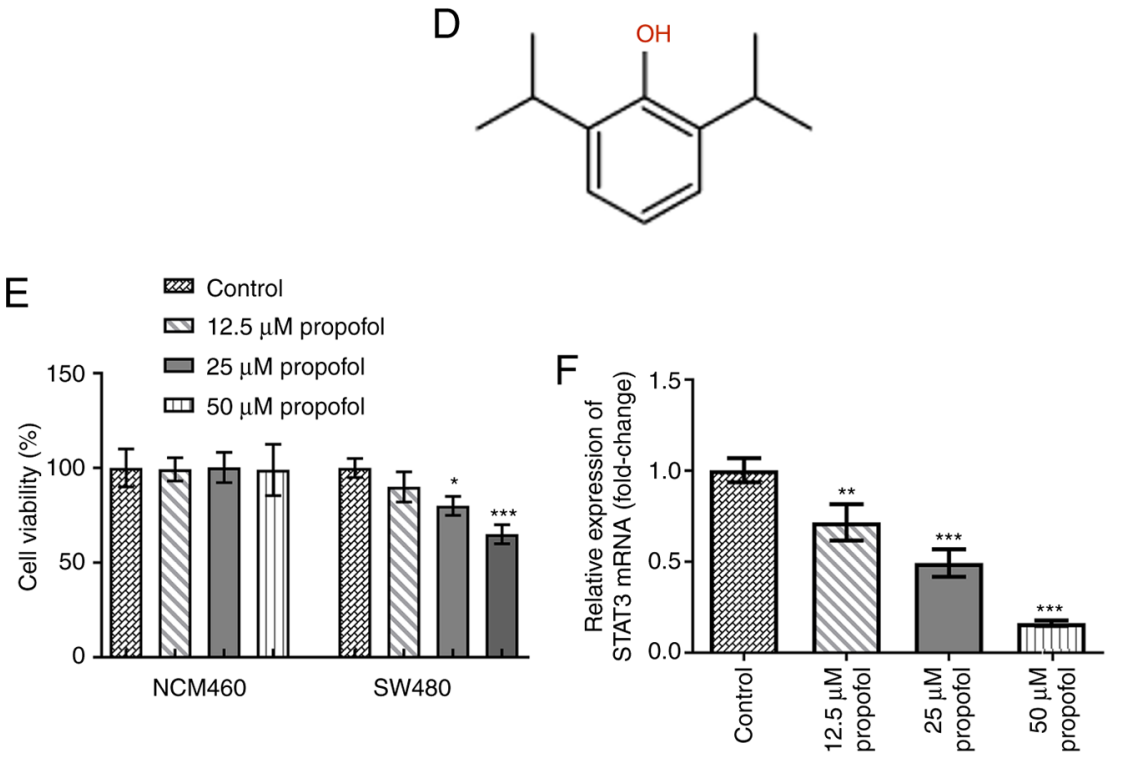

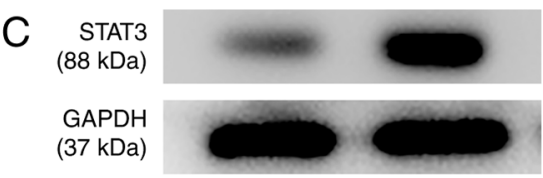

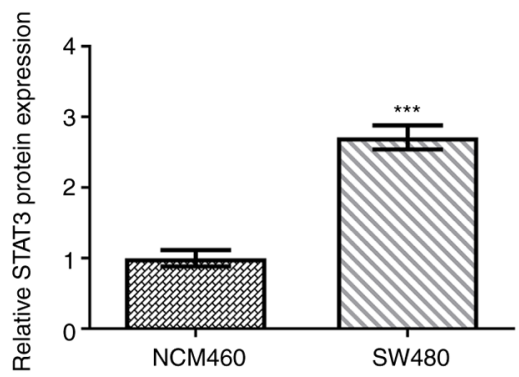

G

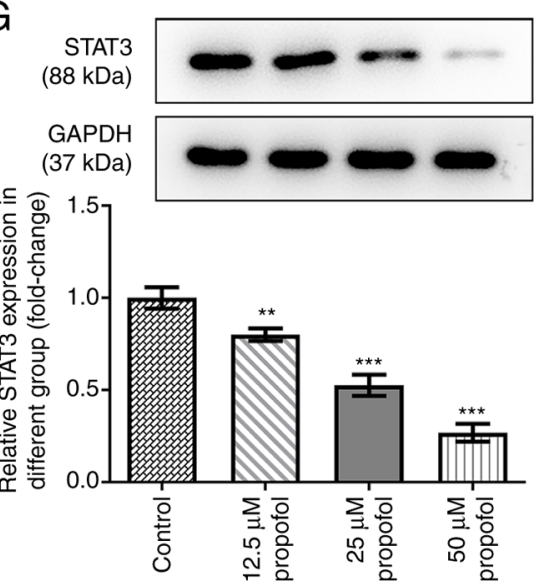

Figure 1. Effect of propofol on cell viability and STAT3 expression in SW480 cells. (A) The mRNA level of STAT3 in colorectal cancer tissues and adjacent control normal tissues after being normalized to the control group $(n=12)$, as detected by RT-qPCR. The (B) mRNA and (C) protein expression of STAT3 in SW480 cells and human intestinal epithelial cells (NCM460) was detected by RT-qPCR and western blotting, respectively. (D) The chemical structure of propofol. (E) The cell viability of NCM460 and SW480 cells exposed to $0,12.5,25$ and $50 \mu \mathrm{m}$ propofol for $48 \mathrm{~h}$ was detected by MTT assay. The (F) mRNA and $(\mathrm{G})$ protein expression of STAT3 in SW480 cells exposed to 0, 12.5, 25 and $50 \mu \mathrm{m}$ propofol for $48 \mathrm{~h}$ was detected by RT-qPCR and western blotting, respectively. ${ }^{*} \mathrm{P}<0.05,{ }^{* *} \mathrm{P}<0.01$ and ${ }^{* * * *} \mathrm{P}<0.001$ vs. control or NCM460 group. $\mathrm{n}=3$. STAT3, signal transducer and activator of transcription 3 ; RT-qPCR, reverse transcription quantitative PCR.

proteins involved in cell proliferation, including Ki67 and PCNA, while STAT3 overexpression partially recovered the expression levels of these proteins (Fig. 2E).

Propofol induces CRC cell ferroptosis, which is blocked by STAT3 overexpression. Finally, the levels of ferroptosis in CRC cells following propofol stimulation were evaluated. Propofol treatment significantly increased TBARS (Fig. 3A), total cellular iron (Fig. 3B), Fe ${ }^{2+}$ (Fig. 3C) and ROS (Fig. 3D) levels. Notably, the content of ROS in propofol group was markedly increased compared with that in the control group (Fig. 3E). In addition, the protein expression levels of $\mathrm{CHAC1}$ and PTGS2 were significantly upregulated, while those of GPX4 were significantly downregulated by propofol treatment (Fig. 4A-D). However, all these effects were markedly weakened by the overexpression of STAT3 (Figs. 3 and 4).

\section{Discussion}

To the best of our knowledge, the causes of the high incidence and mortality rates in CRC remain undetermined. Surgical tumor resection is currently the main clinical treatment method for CRC (21). However, surgery can promote cancer metastasis, as the procedure allows cancer cells to escape and enter the circulatory system, which represents a significant challenge in the effective treatment of CRC that needs to be resolved urgently (7). It has been reported that anesthetics can improve the long-term outcome of patients after surgery, particularly with regard to recurrence and metastasis (22). Accumulating evidence has demonstrated that propofol could inhibit cell proliferation in different types of cancer, including CRC. However, to the best of our knowledge, the specific molecular mechanisms underlying the effects of propofol on the development and progression of $\mathrm{CRC}$ remain to be determined. The results of the present study demonstrated that propofol treatment significantly promoted the ferroptosis and inhibited the proliferation of CRC cells, which suggested that propofol may play a tumor suppressive role in CRC.

Although the research is currently limited to in vitro and animal model experiments, a number of small molecular ferroptosis inducers (e.g., erastin, sorafenib and sulfasalazine) have shown beneficial antitumor effects (23), 
A

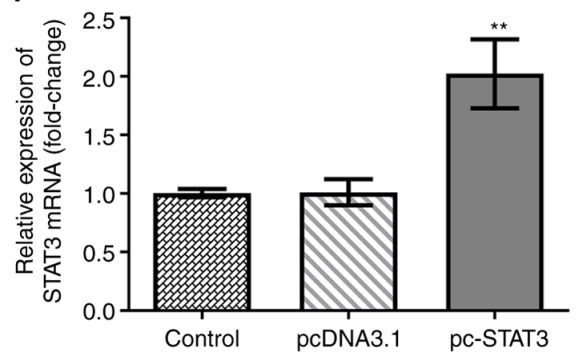

C

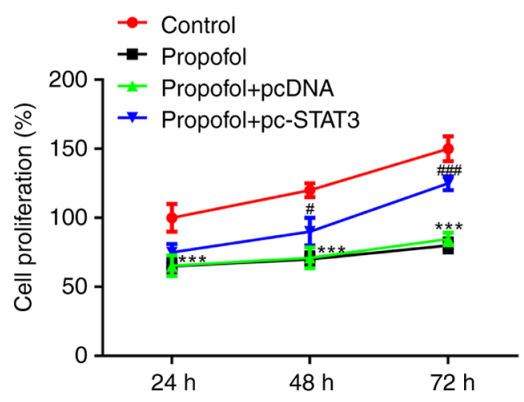

$\mathrm{E}$
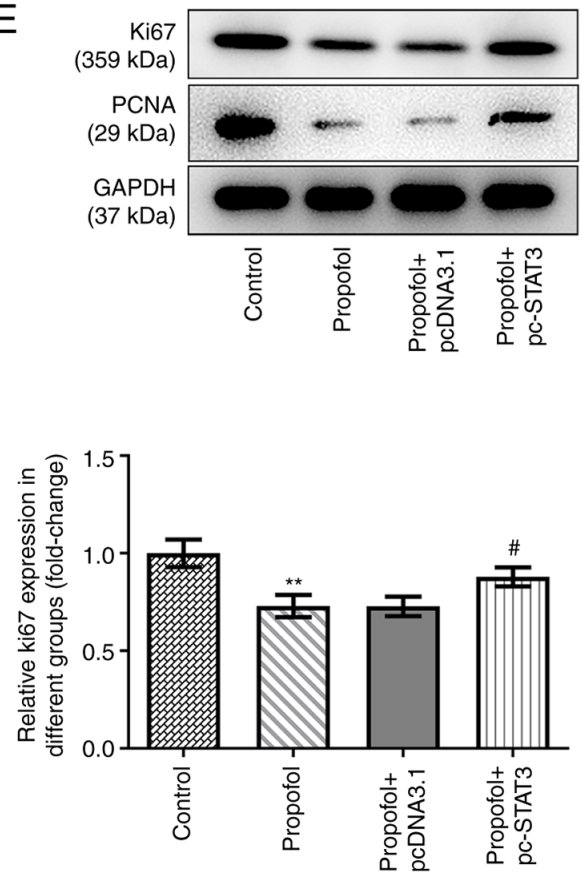

B
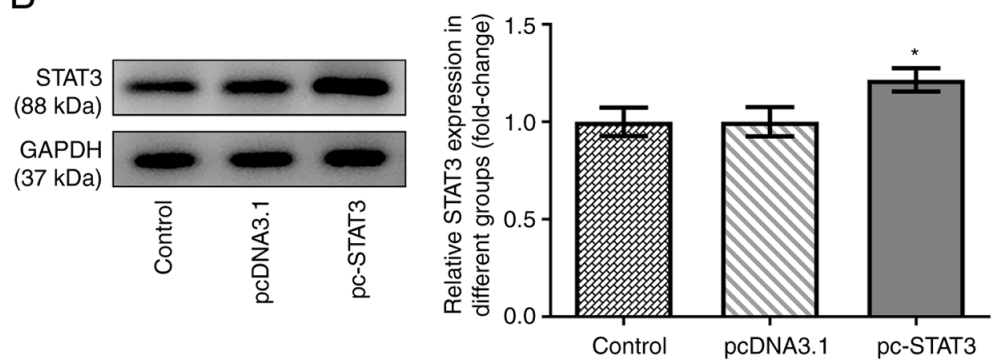

D
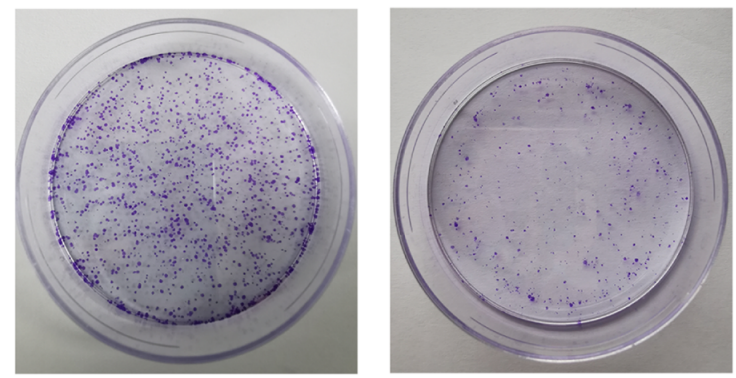

Control

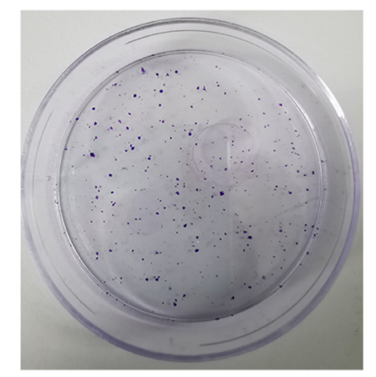

Propofol+pcDNA3.1

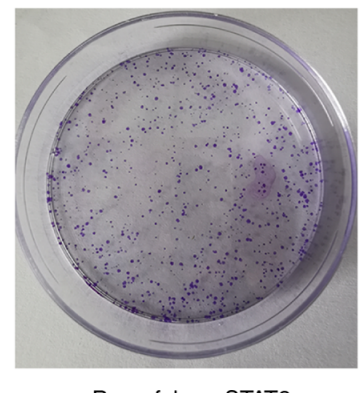

Propofol+pc-STAT3

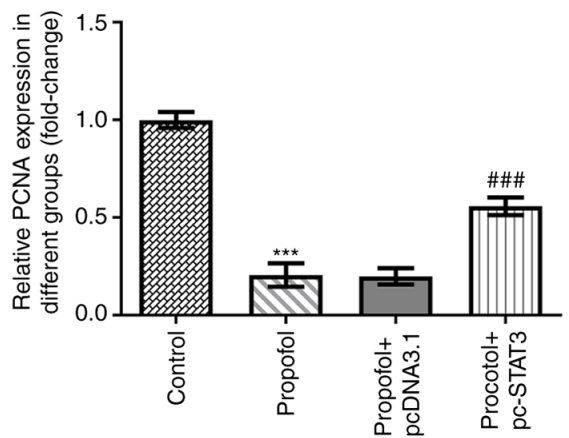

Figure 2. Propofol inhibits colorectal cancer cells proliferation and colony formation, but STAT3 overexpression decreases this effect. The (A) mRNA and (B) protein expression of STAT3 in control SW480 cells and cells transfected with pcDNA3.1 empty vector or pcDNA3.1-STAT3 was detected by RT-qPCR and western blotting, respectively. ${ }^{*} \mathrm{P}<0.05$ and ${ }^{* *} \mathrm{P}<0.01$ vs. pcDNA3.1. (C) The cell viability of control SW480 cells and cells transfected with pcDNA3.1 empty vector or pcDNA3.1-STAT3 at 24, 48 and $72 \mathrm{~h}$ post-transfection was detected by MTT assay. (D) Representative colony formation results of SW480 cells in different groups were determined by colony formation assay. (E) Representative blots and quantitative analysis for Ki67 and PCNA protein expression in SW480 cells in different groups, as detected by western blotting. ${ }^{\text {} *} \mathrm{P}<0.01$ and ${ }^{* * * *} \mathrm{P}<0.001$ vs. control. ${ }^{*} \mathrm{P}<0.05$ and ${ }^{\# \# \#} \mathrm{P}<0.001$ vs. propofol + pcDNA3.1. $\mathrm{n}=3$. STAT3, signal transducer and activator of transcription 3; RT-qPCR, reverse transcription quantitative PCR.

and some anticancer drugs approved by the US Food and Drug Administration have been identified as inducers of ferroptosis (24-26). For example, sorafenib was discovered to increase the levels of lipid oxidation in hepatocellular carcinoma, which led to cell death. Notably, administration of a specific inhibitor of ferroptosis, ferrostatin-1, prevented this effect, while inhibitors of apoptosis and necrosis were unable to prevent this effect (27). In pancreatic cancer, activation of the heat shock protein A5 (HSPA5)/GPX4 signaling pathway induced resistance to gemcitabine, while the knockdown of HSPA5 or GPX4 reversed this drug resistance. Ferroptosis was discovered to play an important role in this process, as GPX4 is known to decrease the accumulation of lipid-free radicals and prevent the occurrence and development of ferroptosis $(28,29)$. A previous study reported that artemisinin and its derivatives triggered ferroptosis in pancreatic cancer 

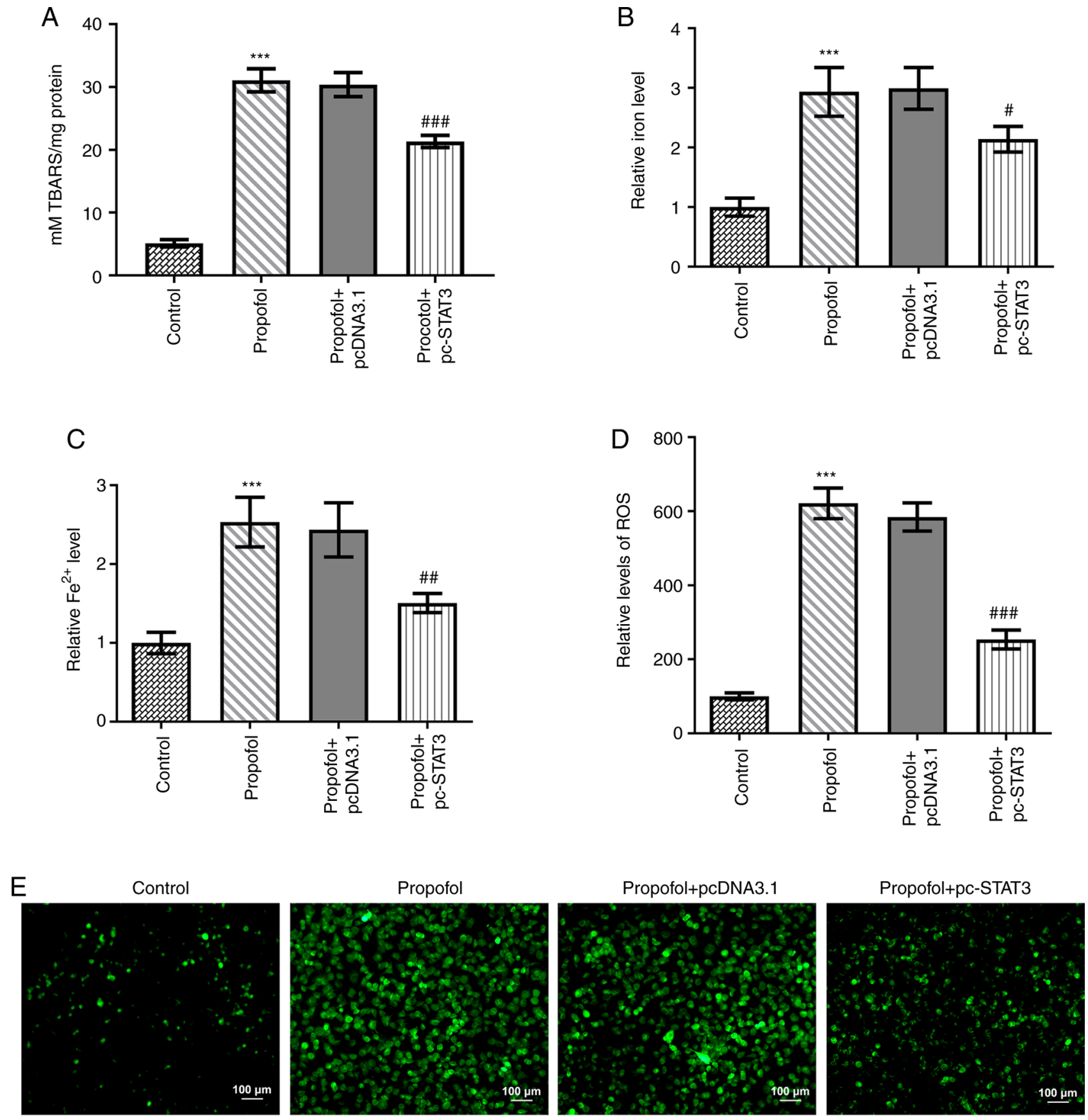

Figure 3. Propofol increases iron, $\mathrm{Fe}^{2+}$ and ROS levels in CRC cells, but STAT3 overexpression decreases this effect. (A) The TBAR level in SW480 cells relative to the control group was detected by TBAR assay kit. The (B) cellular total iron and (C) $\mathrm{Fe}^{2+}$ levels in SW480 cells relative to the control group were detected by iron assay kit. (D) The content of ROS in SW480 cells in different groups were observed using a 2',7'-dichlorofluorescein diacetate-ROS kit. (E) The fluorescence microscopy images of ROS in SW480 cells in different groups. ${ }^{* * *} \mathrm{P}<0.001$ vs. control. ${ }^{*} \mathrm{P}<0.05$, ${ }^{\# \#} \mathrm{P}<0.01$ and ${ }^{\# \#} \mathrm{P}<0.001$ vs. propofol + pcDNA3.1. $\mathrm{n}=3$. TBAR, thiobarbituric acid reactant; ROS, reactive oxygen species; STAT3, signal transducer and activator of transcription 3.

cells with gene mutations in KRAS, but exerted minimal toxic effects on normal cells (30). Proanthocyanidin treatment markedly suppressed the levels of iron, TBAR, acyl-CoA synthase 4 and arachidonate 15-lipoxygenase type $\mathrm{B}$, while upregulating the levels of GSH, GPX4, erythroid 2-related factor 2 and heme oxygenase-1 in cases of spinal cord trauma. To the best of our knowledge, the current study was the first to discover that propofol could promote ferroptosis in CRC cells. Ferroptosis, which is characterized by iron accumulation and lipid peroxidation, is believed to be related to tumor cell
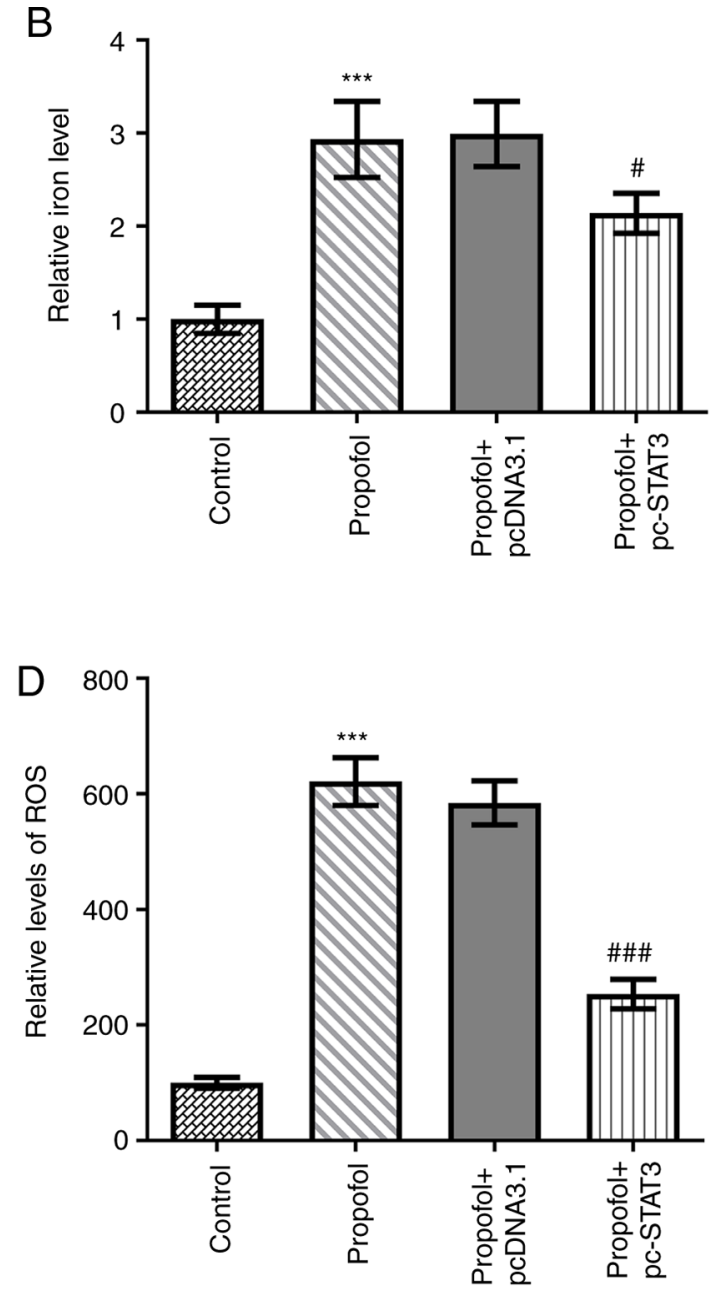
A

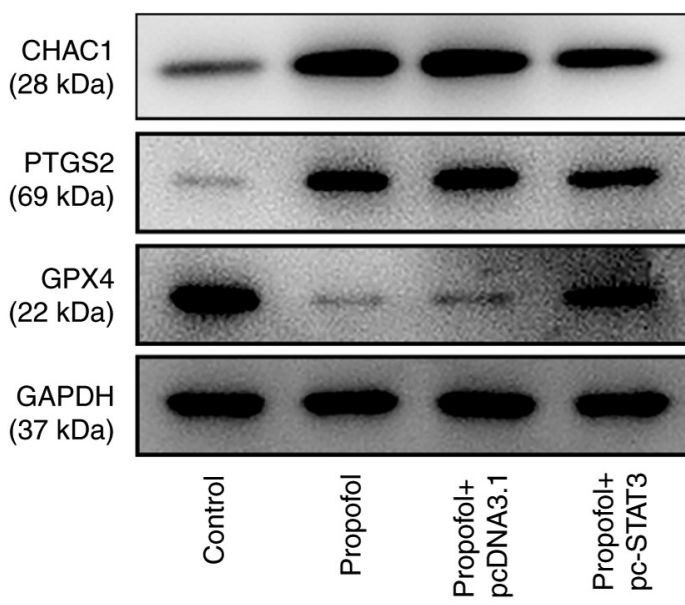

C

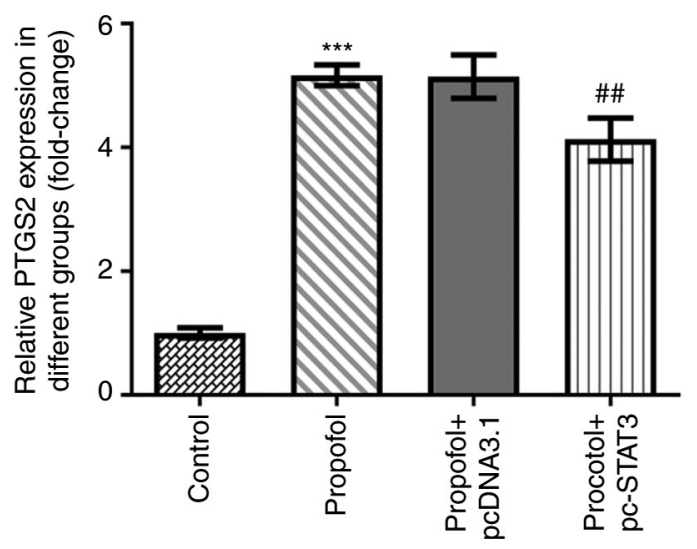

$\mathrm{B}$

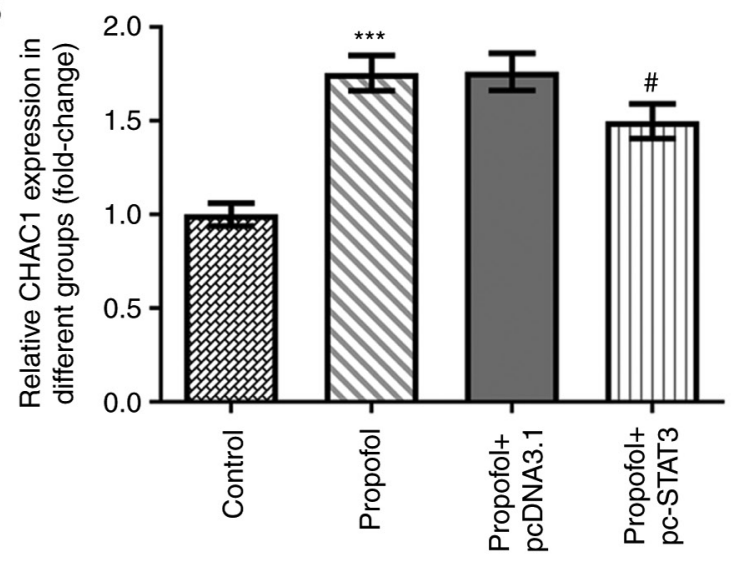

$\mathrm{D}$

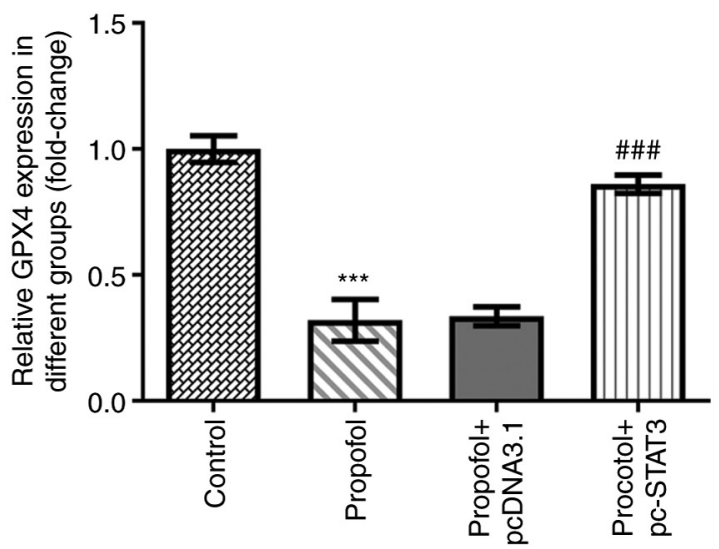

Figure 4. Effect of propofol and STAT3 overexpression on the protein expressions of CHAC1, PTGS2 and GPX4. (A) Representative blots and quantitative analysis for (B) CHAC1, (C) PTGS2 and (D) GPX4 protein expression in SW480 cells in different groups as determined by western blotting. ${ }^{* * *} \mathrm{P}<0.001 \mathrm{vs.} \mathrm{control}$. ${ }^{\#} \mathrm{P}<0.05,{ }^{\# \#} \mathrm{P}<0.01$ and ${ }^{\# \#} \mathrm{P}<0.001$ vs. propofol + pcDNA3.1. $\mathrm{n}=3$. STAT3, signal transducer and activator of transcription 3; CHAC1, cation transport regulator homolog 1; PTGS2, prostaglandin-endoperoxide synthase 2; GPX4, glutathione peroxidase 4 .

GPX4 inactivation promoted lipid peroxide accumulation during ferroptosis in CRC (13). The activating transcription factor 4 (ATF4) branch is the predominant signaling pathway activated by ferroptotic reagents; CHAC1 is downstream of ATF4 and has been demonstrated to promote the degradation of GSH and subsequently induce ferroptosis $(31,32)$. PTGS2 is also induced in cells undergoing ferroptosis (33); however, the exact role of PTGS2 in the ferroptotic cell death cascade remains to be elucidated. Previous studies reported that, inhibiting PTGS2, which is induced by ferroptosis, was an effective method for alleviating cell death $(33,34)$. The present study analyzed the expression levels of GPX4, CHAC1 and PTGS2 in CRC cells following propofol treatment. Consistent with the aforementioned experimental results, propofol treatment markedly downregulated GPX4 expression levels, but upregulated $\mathrm{CHAC} 1$ and PTGS2 expression levels. These data suggested that, consistent with other ferroptosis inducers, propofol may be able to enhance cellular iron levels and ROS accumulation, as well as upregulate the expression levels of CHAC1 and PTGS2, while downregulating those of GPX4.

There are certain limitations to the present study. For example, cell death assays for detecting PI uptake or LDH release were not performed to determine if the decreased cell viability was due to cell death. In addition, experiments using ferroptosis inhibitor (ferrostatin-1) were not conducted to distinguish between ferroptosis and apoptosis. Due to limited funding, not all ferroptosis clarification experiments could be performed. However, the characteristics of ferroptosis, in which GPX4 decreases, PTGS2 increases and $\mathrm{Fe}^{2+}$ increases, were observed and a TBAR lipid peroxidation assay was conducted, which also indicated the presence of ferroptosis.

To further determine the potential underlying mechanism involved in the effects of propofol, the STITCH database was used to identify the regulation of STAT3 by propofol. STAT3 expression is known to be upregulated in the majority of cancer types, including in CRC tissues compared with adjacent control normal tissues. Notably, it has been widely reported that propofol can target STAT3-related signaling pathways, thereby playing a key a role in various diseases. For example, propofol was found to enhance cisplatin-induced cell apoptosis in cervical cancer cells via the EGFR/JAK2/STAT3 signaling pathway (35). Propofol also prevented oxidative stress and apoptosis by regulating iron homeostasis and targeting the JAK/STAT3 signaling pathway in SH-SY5Y cells (36). In addition, a previous study demonstrated that propofol inhibited cell invasion and promoted cell apoptosis 
by regulating the STAT3/HOX transcript antisense RNA axis in CRC (37). The findings of the present study revealed that propofol treatment negatively regulated STAT3 expression in a concentration-dependent manner. Notably, STAT3 has also been suggested to regulate ferroptosis. For example, the administration of small molecules that inhibit STAT3 phosphorylation was reported to increase cellular ROS levels and decrease the GSH/glutathione disulfide ratio (38). In addition, $\alpha 6 \beta 4$ integrin-mediated STAT3 activation was found to promote resistance to ferroptosis (39). Cisplatin-resistant osteosarcoma cells could inhibit ferroptosis after low-dose cisplatin irradiation; however, the administration of ferroptosis agonists and STAT3 inhibitors reactivated ferroptosis, thereby increasing the sensitivity to cisplatin (40). STAT3 overexpression also promoted the migration and invasion of HT-29 cells (41). Activation and phosphorylation of STAT3 could markedly promote the proliferation and metastasis of SW480 cells, and enhance tumorigenesis (42). In one study, the migration and invasion of colon cancer cells was promoted, possibly via the activation of the STAT3 pathway (43). Therefore, it was hypothesized that propofol may promote CRC cell ferroptosis via targeting STAT3. In the present study, STAT3 was overexpressed in CRC cells to investigate whether the effects of propofol on CRC cell proliferation and ferroptosis could be reversed. As expected, the inhibitory effect of propofol on CRC cell proliferation and colony formation, together with the stimulatory effect of propofol on ferroptosis, were all significantly inhibited by STAT3 overexpression. The aforementioned data suggested that propofol may induce CRC cell ferroptosis by downregulating STAT3 expression. To the best of our knowledge, the present study is the first to determine the effect of propofol on ferroptosis in cancer. However, the study conclusions were based on results obtained from an in vitro cell model; therefore, further in vivo experiments are required to verify these findings.

In conclusion, the present study demonstrated that propofol promoted ferroptosis in CRC cells and that the potential mechanism may be dependent on targeting STAT3 expression. Therefore, propofol-induced ferroptosis may represent a promising therapeutic option for CRC.

\section{Acknowledgements}

Not applicable.

\section{Funding}

Not applicable.

\section{Availability of data and materials}

The datasets used and/or analyzed during the current study are available from the corresponding author on reasonable request.

\section{Authors' contributions}

$\mathrm{XZ}$ and FC conceived and designed the study. XZ was responsible for the acquisition analysis and interpretation of data. FC was responsible for manuscript preparation, writing and critical revisions. XZ and FC confirm the authenticity of all the raw data. Both authors have read and approved the manuscript.

\section{Ethics approval and consent to participate}

All the patients provided written, informed consent and the study was approved by the Ethical Committee of Zhongshan Hospital (approval no. B2016-014).

\section{Patient consent for publication}

Not applicable.

\section{Competing interests}

The authors declare that they have no competing interests.

\section{References}

1. Bray F, Ferlay J, Soerjomataram I, Siegel RL, Torre LA and Jemal A: Global cancer statistics 2018: GLOBOCAN estimates of incidence and mortality worldwide for 36 cancers in 185 countries. CA Cancer J Clin 68: 394-424, 2018.

2. Dekker E, Tanis PJ, Vleugels JLA, Kasi PM and Wallace MB: Colorectal cancer. Lancet 394: 1467-1480, 2019.

3. Brody H: Colorectal cancer. Nature 521: S1, 2015.

4. Bateman BT and Kesselheim AS: Propofol as a transformative drug in anesthesia: Insights from key early investigators. Drug Discov Today 20: 1012-1017, 2015

5. Jiang S, Liu Y, Huang L, Zhang F and Kang R: Effects of propofol on cancer development and chemotherapy: Potential mechanisms. Eur J Pharmacol 831: 46-51, 2018.

6. Wu ZF, Lee MS, Wong CS, Lu CH, Huang YS, Lin KT, Lou YS, Lin C, Chang YC and Lai HC: Propofol-based total intravenous anesthesia is associated with better survival than desflurane anesthesia in colon cancer surgery. Anesthesiology 129: 932-941, 2018.

7. Ren YL and Zhang W: Propofol promotes apoptosis of colorectal cancer cells via alleviating the suppression of IncRNA HOXA11-AS on miRNA let-7i. Biochem Cell Biol 98: 90-98, 2020.

8. Hickman JA: Apoptosis induced by anticancer drugs. Cancer Metastasis Rev 11: 121-139, 1992.

9. Mohammad RM, Muqbil I, Lowe L, Yedjou C, Hsu HY, Lin LT, Siegelin MD, Fimognari C, Kumar NB and Dou QP: Broad targeting of resistance to apoptosis in cancer. Semin Cancer Biol 35: S78-S103, 2015.

10. Hassannia B, Vandenabeele P and Berghe TV: Targeting ferroptosis to iron out cancer. Cancer Cell 35: 830-849, 2019.

11. Mou Y, Wang J, Wu J, He D, Zhang C, Duan C and Li B: Ferroptosis, a new form of cell death: Opportunities and challenges in cancer. J Hematol Oncol 12: 34, 2019.

12. Xu X, Zhang X, Wei C, Zheng D, Lu X, Yang Y, Luo A, Zhang K, Duan X and Wang Y: Targeting SLC7A11 specifically suppresses the progression of colorectal cancer stem cells via inducing ferroptosis. Eur J Pharm Sci 152: 105450, 2020.

13. Sui X, Zhang R, Liu S, Duan T, Zhai L, Zhang M, Han X, Xiang Y, Huang X, Lin H and Xie T: RSL3 drives ferroptosis through GPX4 inactivation and ROS production in colorectal cancer. Front Pharmacol 9: 1371, 2018.

14. Zhang L, Liu W, Liu F, Wang Q, Song M, Yu Q, Tang K, Teng T, Wu D, Wang X, et al: IMCA induces ferroptosis mediated by SLC7A11 through the AMPK/mTOR pathway in colorectal cancer. Oxid Med Cell Longev 2020: 1675613, 2020.

15. Liang WZ, Jan $\mathrm{CR}$ and $\mathrm{Lu} \mathrm{CH}$ : Investigation of 2,6-diisopropylphenol (propofol)-evoked $\mathrm{Ca} 2+$ movement and cell death in human glioblastoma cells. Toxicol In Vitro 26: 862-871, 2012.

16. Wang H, Zhao L, Wu J, Hong J and Wang S: Propofol induces ROS-mediated intrinsic apoptosis and migration in triple-negative breast cancer cells. Oncol Lett 20: 810-816, 2020.

17. Yu H, Lee H, Herrmann A, Buettner R and Jove R: Revisiting STAT3 signalling in cancer: New and unexpected biological functions. Nat Rev Cancer 14: 736-746, 2014. 
18. Zhou B, Liu J, Kang R, Klionsky DJ, Kroemer G and Tang D Ferroptosis is a type of autophagy-dependent cell death. Semin Cancer Biol 66: 89-100, 2019.

19. Zhang Y, Zhu M, Mohan SK and Hao Z: Crocin treatment promotes the oxidative stress and apoptosis in human thyroid cancer cells FTC-133 through the inhibition of STAT/JAK signaling pathway. J Biochem Mol Toxicol 35: e22608, 2021.

20. Livak KJ and Schmittgen TD: Analysis of relative gene expression data using real-time quantitative PCR and the 2(-Delta Delta C(T)) method. Methods 25: 402-408, 2001.

21. Xu Z: Surgical treatment of colorectal cancer. Chin Oncol 23: 389-398, 2013 (In Chinese).

22. Cassinello F, Prieto I, del Olmo M, Rivas S and Strichartz GR: Cancer surgery: How may anesthesia influence outcome? J Clin Anesth 27: 262-272, 2015.

23. Liang $\mathrm{C}$, Zhang $\mathrm{X}$, Yang $\mathrm{M}$ and Dong $\mathrm{X}$ : Recent progress in ferroptosis inducers for cancer therapy. Adv Mater 31: e1904197, 2019.

24. Trujillo-Alonso V, Pratt EC, Zong H, Lara-Martinez A, Kaittanis C, Rabie MO, Longo V, Becker MW, Roboz GJ, Grimm J and Guzman ML: FDA-approved ferumoxytol displays anti-leukaemia efficacy against cells with low ferroportin levels. Nat Nanotechnol 14: 616-622, 2019.

25. Lo M, Ling V, Low C, Wang YZ and Gout PW: Potential use of the anti-inflammatory drug, sulfasalazine, for targeted therapy of pancreatic cancer. Curr Oncol 17: 9-16, 2010.

26. Zhang Y, Shi J, Liu X, Feng L, Gong Z, Koppula P, Sirohi K, Li X, Wei Y, Lee H, et al: BAP1 links metabolic regulation of ferroptosis to tumour suppression. Nat Cell Biol 20: 1181-1192, 2018.

27. Sun X, Ou Z, Chen R, Niu X, Chen D, Kang R and Tang D: Activation of the $\mathrm{p} 62$-Keap1-NRF2 pathway protects against ferroptosis in hepatocellular carcinoma cells. Hepatology 63 : 173-184, 2016.

28. Zhu S, Zhang Q, Sun X, Zeh HJ III, Lotze MT, Kang R and Tang D: HSPA5 regulates ferroptotic cell death in cancer cells. Cancer Res 77: 2064-2077, 2017.

29. Tang H, Chen D, Li C, Zheng C, Wu X, Zhang Y, Song Q and Fei W: Dual GSH-exhausting sorafenib loaded manganese-silica nanodrugs for inducing the ferroptosis of hepatocellular carcinoma cells. Int J Pharm 572: 118782, 2019.

30. Wang K, Zhang Z, Wang M, Cao X, Qi J, Wang D, Gong A and Zhu H: Role of GRP78 inhibiting artesunate-induced ferroptosis in KRAS mutant pancreatic cancer cells. Drug Des Dev Ther 13: 2135-2144, 2019

31. Xu T, Ding W, Ji X, Ao X, Liu Y, Yu W and Wang J: Molecular mechanisms of ferroptosis and its role in cancer therapy. J Cell Mol Med 23: 4900-4912, 2019.
32. Dixon SJ, Patel DN, Welsch M, Skouta R, Lee ED, Hayano M, Thomas AG, Gleason CE, Tatonetti NP, Slusher BS and Stockwell BR: Pharmacological inhibition of cystine-glutamate exchange induces endoplasmic reticulum stress and ferroptosis. Elife 3: e02523, 2014.

33. Xiao X, Jiang Y, Liang W, Wang Y, Cao S, Yan H, Gao L and Zhang L: miR-212-5p attenuates ferroptotic neuronal death after traumatic brain injury by targeting Ptgs2. Mol Brain 12: 78, 2019.

34. Li Q, Han X, Lan X, Gao Y, Wan J, Durham F, Cheng T, Yang J, Wang Z, Jiang C, et al: Inhibition of neuronal ferroptosis protects hemorrhagic brain. JCI Insight 2: e90777, 2017.

35. Li H, Lu Y, Pang Y, Li M, Cheng X and Chen J: Propofol enhances the cisplatin-induced apoptosis on cervical cancer cells via EGFR/JAK2/STAT3 pathway. Biomed Pharmacother 86: 324-333, 2017.

36. Zhang Y, Zuo Y, Li B, Xie J, Ma Z, Thirupathi A, Yu P, Gao G, Shi M, Zhou C, et al: Propofol prevents oxidative stress and apoptosis by regulating iron homeostasis and targeting JAK/STAT3 signaling in SH-SY5Y cells. Brain Res Bull 153: 191-201, 2019.

37. Zhang YF, Li CS, Zhou Y and Lu XH: Effects of propofol on colon cancer metastasis through STAT3/HOTAIR axis by activating WIF-1 and suppressing wnt pathway. Cancer Med 9: $1842-1854,2020$

38. Hu S, Sechi M, Singh PK, Dai L, McCann S, Sun D, Ljungman M and Neamati N: A novel redox modulator induces a GPX4-mediated cell death that is dependent on iron and reactive oxygen species. J Med Chem 63: 9838-9855, 2020.

39. Brown CW, Amante JJ, Goel HL and Mercurio AM: The $\alpha 6 \beta 4$ integrin promotes resistance to ferroptosis. J Cell Biol 216: 4287-4297, 2017.

40. Liu Q and Wang K: The induction of ferroptosis by impairing STAT3/Nrf2/GPx4 signaling enhances the sensitivity of osteosarcoma cells to cisplatin. Cell Biol Int 43: 1245-1256, 2019.

41. Zhang GY, Yang WH and Chen Z: Upregulated STAT3 and RhoA signaling in colorectal cancer (CRC) regulate the invasion and migration of CRC cells. Eur Rev Med Pharmacol Sci 20: 2028-2037, 2016.

42. Zhao FL and Qin CF: EGF promotes HIF-1 $\alpha$ expression in colorectal cancer cells and tumor metastasis by regulating phosphorylation of STAT3. Eur Rev Med Pharmacol Sci 23: 1055-1062, 2019.

43. Zhang WJ, Hu CG, Luo HL and Zhu ZM: Activation of P2x7 receptor promotes the invasion and migration of colon cancer cells via the STAT3 signaling. Front Cell Dev Biol 8: 586555 , 2020.

cc) (i) () $\odot$ This work is licensed under a Creative Commons c) Attribution-NonCommercial-NoDerivatives 4.0 International (CC BY-NC-ND 4.0) License. 